\title{
Labyrinthe
}

25 | 2006 (3)

La Bande dessinée : ce qu'elle dit, ce qu'elle montre

\section{DAVID B., le sommeil de la raison}

\section{Renaud Pasquier}

\section{(2) OpenEdition}

Journals

\section{Édition électronique}

URL : http://journals.openedition.org/labyrinthe/1437

DOI : $10.4000 /$ labyrinthe. 1437

ISSN : 1950-6031

\section{Éditeur}

Hermann

\section{Édition imprimée}

Date de publication : 1 décembre 2006

Pagination : 107-117

ISBN : 2-9526131-2-5

Référence électronique

Renaud Pasquier, « DAVID B., le sommeil de la raison », Labyrinthe [En ligne], 25 | 2006 (3), mis en ligne le 14 décembre 2006, consulté le 10 décembre 2020. URL : http://journals.openedition.org/labyrinthe/ 1437 ; DOI : https://doi.org/10.4000/labyrinthe.1437

Ce document a été généré automatiquement le 10 décembre 2020.

Propriété intellectuelle 


\title{
DAVID B., le sommeil de la raison
}

\author{
Renaud Pasquier
}

El sueno de la razon produce monstruos /

Le sommeil de la raison engendre des monstres

FRANCISCO GOYA, 1797.

Je dessine des monstres, je produis des monstres. DAVID. B., l'Ascension du Haut Mal, tome VI, p.49.

1 «L'Ascension du Haut Mal, chef-d'œuvre de l'autobiographie en bande dessinée »: le jugement, fort répandu, laisse perplexe. Un sentiment en aucun cas suscité par l'éloge, mille et mille fois mérité, mais par la catégorie d'autobiographie. Certes, Pierre-François Beauchard, devenu David, puis David B., s'y met bien en scène dans un récit qui retrace sa vie, de son enfance, dans les années 60 , à aujourd'hui : on peut s'en contenter pour employer le terme.

2 Un simple regard sur les couvertures des six tomes suffit pourtant à contrarier cette rapide identification générique. Six images, déjà une "bande dessinée » en soi : tandis qu'en arrière-plan, le noir de silhouettes fantastiques et inquiétantes, toujours plus nombreuses, recouvre peu à peu le jaune du fond (représentation concrète de «l'ascension » du titre ?), les deux figures masculines du premier plan vieillissent peu à peu, leurs sourires disparaissent, une gravité triste envahit leurs traits. Non pas une figure, mais deux : le préfixe de l'autobiographie est mis à mal. David (l'identité, le même, le moi) n'est pas seul au cœur de l'Ascension du Haut Mal, il passe même au second plan derrière Jean-Christophe, son frère malade, victime d'une forme d'épilepsie particulièrement aiguë, le «Haut Mal» du titre, qui bouleverse la vie de la famille Beauchard. 

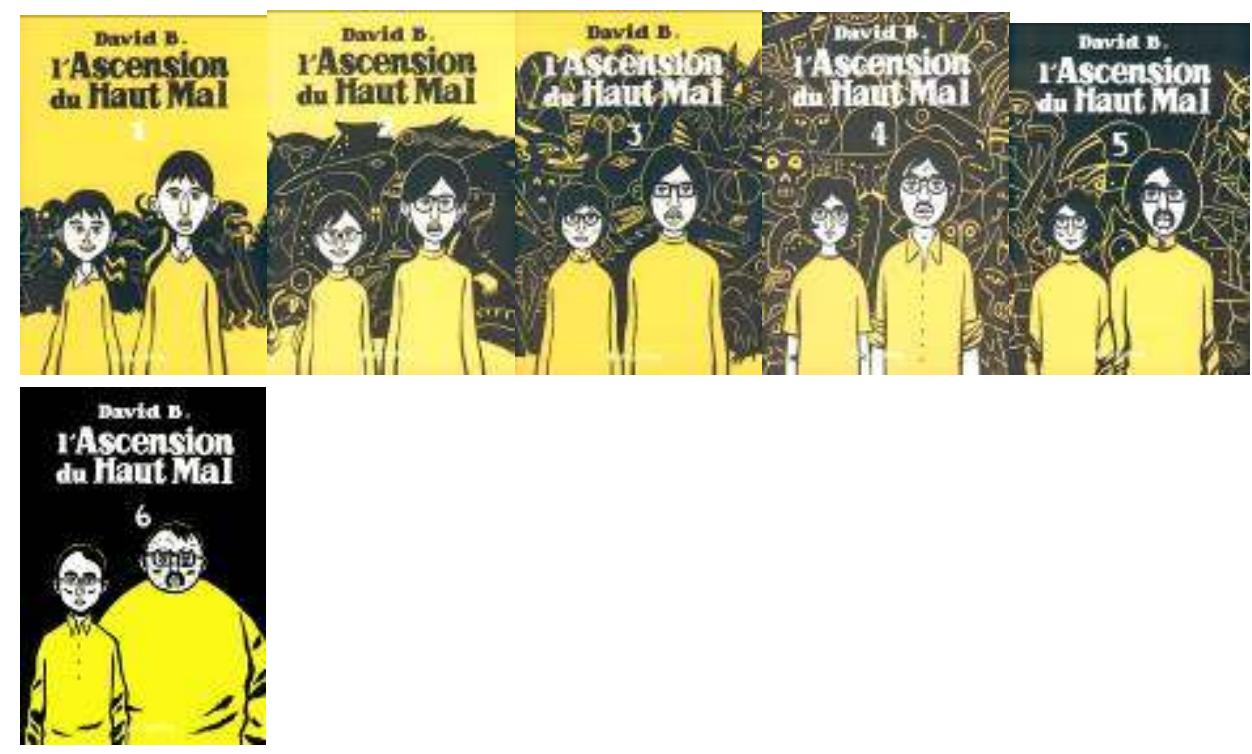

l'Ascension du Haut Mal, David B. (C) L'Association.

Le frère, ce quasi-même dont l'altérité n'est que plus sensible; la maladie, soit l'autre en soi, qui plus est mystérieuse et incurable: osera-t-on alors parler d'altrobiographie? Pourquoi pas, si l'on aime les néologismes... Tâchons surtout de mieux cerner cet " autre ", qui en l'état ne suffit pas, concept passe-partout et du coup guère opératoire pour aborder l'œuvre. Rappelons comment David B. énonce son projet: «Je voulais raconter trois choses : la maladie de mon frère, la construction de mon imaginaire, et la vie de ma famille, avec les parents, les grands-parents, etc. $»^{1}$ Trois éléments à ne pas mettre sur le même plan, «le Haut Mal» de Jean-Christophe demeurant la clef de voûte de l'ensemble: c'est sous son influence - au sens quasi-magique du terme - que se développe l'onirisme angoissé de David B. $^{2}$; c'est aussi lui qui oblige à relire l'histoire d'une famille marquée par son signe, un passé de légendes et superstitions paysannes, mais aussi de guerres absurdes. Le "Haut Mal» ne désigne alors plus seulement l'épilepsie, mais toute une série de maux, toute une série d'«autres" menaçants et invisible. David B. leur donne un nom, un visage : ceux du Monstre.

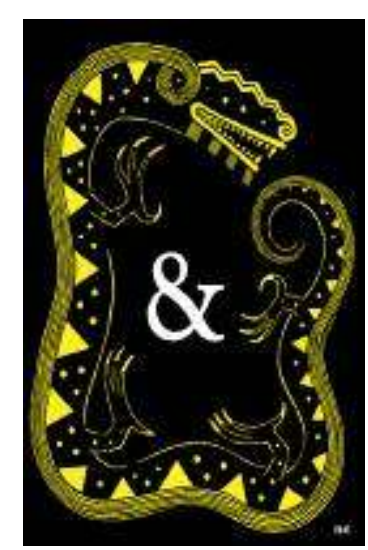

I'Ascension du Haut Mal, David B. ( L L'Association.

Qu'est-ce qu'un monstre? «Être, animal fantastique (des légendes, mythologies); cf. chimère, dragon, etc. » nous dit le dictionnaire. Les monstres sont engendrés par «le sommeil de la raison ", nous prévient aussi un célèbre tableau de Goya. David B. a publié deux recueils de récits de rêves ${ }^{3}$ (ou plutôt de cauchemars), et tous ses travaux manifestent son goût 
pour les contes, mythes et légendes de tous pays. Comme s'il s'agissait de prendre le relais d'une raison défaillante (ou endormie) face au péril ; l'Ascension naît ainsi de «cette rage qui me prenait quand on me disait qu'on n'avait pas d'explication pour ce phénomène, ni de solution crédible et efficace à proposer. ${ }^{4}$ » Représenter le (haut) mal, c'est une manière de le combattre: mais comment le figurer? Comment dessiner l'épilepsie s'emparant soudain de son frère? Le monstrueux reptile (qui n'apparaît d'ailleurs que dans le deuxième volume, puis devient peu à peu omniprésent, au fur et à mesure que «l'ascension » se poursuit) est la réponse à cette double question, et ne doit pas être regardé comme une simple figure conventionnelle du mal. Certes, il exprime l'impuissance de la raison (en accord avec le terme «antéscientifique » de "haut mal » pour désigner l'épilepsie), la nécessité de recourir à un imaginaire fantastique pour appréhender la maladie. Mais il est aussi, par ses courbes apparemment infinies, la solution graphique la plus exacte pour figurer le corps de Jean-Christophe secoué par la crise.
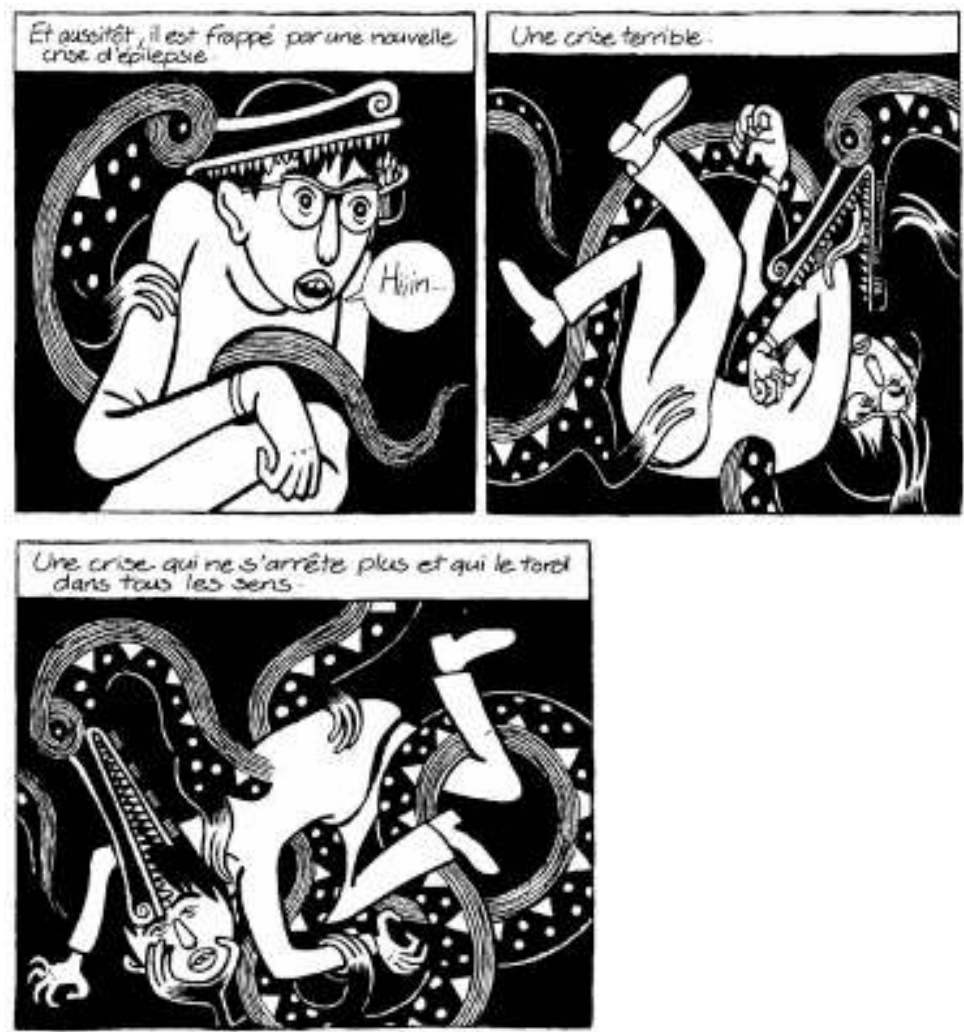

l'Ascension du Haut Mal, David B. (C) L'Association.

Ainsi fait-il la jonction entre deux versants de l'Ascension : le récit fantasmagorique et la chronique très vraie des malheurs d'une famille française à la fin $\mathrm{du} \mathrm{xx}^{\mathrm{e}}$ siècle. Entre fable et Histoire, on glisse d'une définition du monstre à l'autre: "Personne effrayante par son caractère, son comportement (spécialement sa méchanceté). C'est un monstre de cruauté. »Pas seulement des créatures fantastiques, donc, mais aussi des bourreaux, des dictateurs sanguinaires, des conquérants meurtriers : comme un équivalent collectif à la maladie de Jean-Christophe, d'autres produits du "sommeil de la raison». Hitler est une figure récurrente (parfois symbolisé par la croix gammée) de l'Ascension, souvent liée à l'ésotérisme et la magie, ou bien présentée comme fantasme inquiétant de Jean- 
Christophe. Où se confirmerait le recours à la Bande dessinée comme exorcisme, moyen de circonscrire les monstres qui échappent aux démarches scientifiques et rationnelles.

L'affaire est pourtant plus complexe. Il ne s'agit pas seulement de débusquer et combattre les monstres. Il y a chez David B. une fascination assumée pour ceux-ci, voire une sympathie revendiquée. David s'invente pour confidents imaginaires des créatures de cauchemar, tandis qu'avec sa famille, il entreprend un étrange voyage chez les guérisseurs, voyants, charlatans, illuminés de tout poil que ses parents consultent dans l'espoir, toujours déçu, de guérir leur fils aîné. Où le motif du monstre va jusqu'à imposer la forme même du récit; la structure de répétition (narrative et graphique) procède certes de la réalité des faits (l'éternel recommencement des vaines thérapies) et de l'obsession qui en découle pour la famille Beauchard. Mais ce ressassement correspond aussi à la présentation requise par le monstre, à savoir la galerie. La narration s'infléchit bien souvent en une série de portraits, plutôt que d'événements, ceux des excentriques personnages rencontrés par la famille Beauchard, de telle ou telle figure historique non moins excentrique (Swedenborg, Raymond Abellio), ou des aïeux de David. Autant de digressions, de micro-récits, ou plutôt de planches qui reçoivent une quasi-autonomie, la "voix-off» des récitatifs, celle de l'auteur, étant alors presque seule garante de la «solidarité iconique »- pour reprendre l'expression de Thierry Groensteen. La tendance s'accuse au fur et à mesure de l'ascension, et le tome six relève parfois plus du recueil de visions ${ }^{5}$ que du récit linéaire et continu : les détails y prolifèrent, la structure des cases est souvent ignorée ou bien devient un élément à part entière du dessin (voir notamment $p$. 41). "Galerie », " portrait », on est bien sûr dans l'univers de la peinture, et sans doute y a-t-il une tendance picturale du très littéraire David B., mais ce n'est pas là le plus important, me semble-t-il. La galerie évoque plutôt le monde médical, et le passage en revue de tous les cas possibles d'une même pathologie, photos à l'appui. Des phénomènes pathologiques aux monstres de foire, il n'y a qu'un pas : ce n'est pas au musée que l'on trouvera une galerie des monstres, mais au cirque - et l'on connaît le goût de David B. pour le cirque. « La maison est comme un chapiteau de cirque. Des artistes de l'irrationnel viennent y faire leur numéro » (tome V, p.32). «Monstre », en l'occurrence, se dira plus adéquatement en langue anglaise: freak, créature marginale, rejetée par le groupe. Impossible bien sûr de ne pas songer au film de Tod Browning ${ }^{6}$ : comme lui, David B. se place du côté des freaks, et non en observateur curieux ou dégoûté. Jean-Christophe est un freak, sa famille le devient à sa suite, et toute cette humanité bizarre qu'ils rencontrent au gré de leurs pérégrinations est constituée d'une infinité de freaks....

«Nous avions basculé dans un univers qui n'était pas le monde naturel, celui dans lequel vivaient les autres. Et au terme de ce basculement, c'étaient nos amis qui étaient des monstres...Le monde "normal" ne pouvait rien pour nous, alors autant se tourner vers les forces mystérieuses. ${ }^{7}$

7 Dans la tératologie, ou plutôt tératographie (car le logos y est plutôt mis à mal) de David B., faut-il alors distinguer les " gentils » freaks et les «méchants » Monstres? Ce serait trop simple, on va le montrer. Dans le jeu de correspondances et d'articulations entre les vies individuelles et l'Histoire collective, Jean-Christophe est comparé aux freaks par excellence, dans le sens le plus tragique du terme, que sont les Juifs victimes des nazis :

«La question du Génocide, de la Shoah, est pour moi automatiquement reliée à la maladie de Jean-Christophe. À le voir "mourir" quotidiennement, on finit par avoir l'impression d'avoir assisté à un grand nombre de morts, donc à une sorte de massacre... Dans cet acharnement de la maladie, il y a quelque chose comme une volonté de détruire.... ${ }^{8}$ 
Ce rapprochement est mis en image dans le quatrième tome, où à deux pages de distances se suivent la représentation d'un Juif victime des camps, dans la posture même que David B. confère d'ordinaire à son frère en pleine crise, puis la vision, en trois cases, de toutes les « morts » de Jean-Christophe depuis le début de sa maladie. Le rapprochement repose donc sur une perception très concrète, sur une métamorphose du corps, mais aussi sur le statut de l'événement : le traumatisme subi par la famille Beauchard devant l'inexplicable horreur serait une projection, à une échelle très réduite, du choc que représente pour l'humanité entière l'extermination des Juifs par les nazis. Primo Levi, reprenant le titre de Goya, ne parlait-il pas du Lager comme du « plus menaçant des monstres engendrés par le sommeil de la raison ${ }^{9} »$ ?
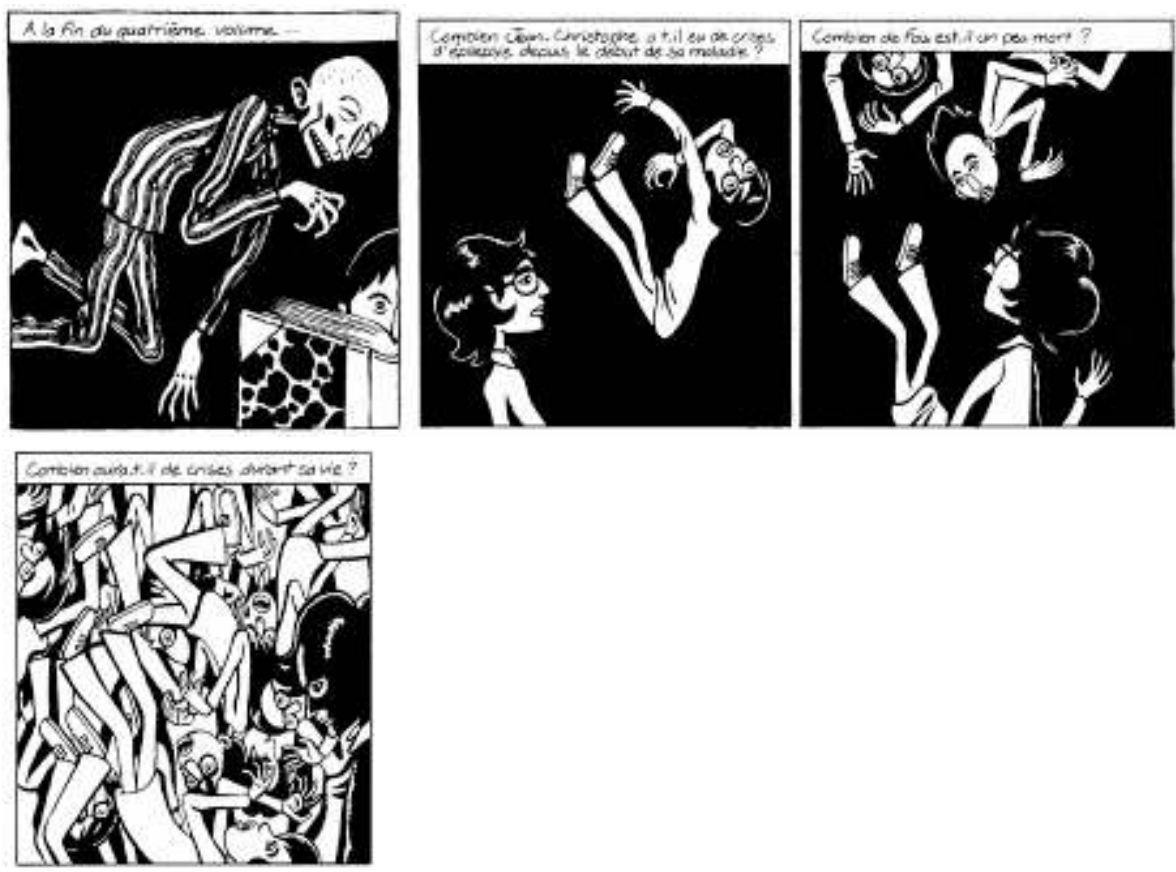

l'Ascension du Haut Mal, David B. (C) L'Association.

Mais au-delà de ces analogies, l'évocation du génocide perpétré par les nazis joue, dans le contexte de l'Ascension, un rôle déterminant. Les deux pôles du Monstre y sont présentes sur un mode superlatif: le Juif, c'est-à-dire le freak, la victime ; Hitler, l'Ennemi, le Monstre sanguinaire. Or Jean-Christophe, placé sur le même plan que le Juif, a fait d'Hitler son idole : lui, l'homme de la ligne courbe, désarticulé par les convulsions de la crise, rêve des lignes droites de la croix gammée et des bras tendus; de fait le freak peut se faire Monstre, devenir un danger pour les siens, agresser plusieurs fois son père et son frère. Or ce dernier, Pierre-François, adopte le prénom « David " par fascination pour les Juifs et leur culture (jamais démentie, en témoigne son évocation des années passées rue des Rosiers). Mais lui-même est souvent envahi par des désirs de meurtre à l'égard de Jean-Christophe, qu'il peut aussi harceler, sûr, en définitive, de sa supériorité. 


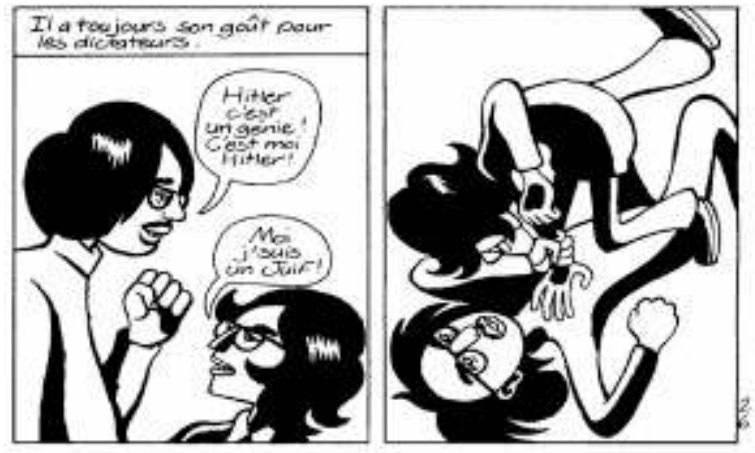

I'Ascension du Haut Mal, David B. (c) L'Association.

En somme l'écart entre «bon » et « mauvais » monstre est brouillée, et pas seulement par les deux frères : la mutation du freak en Monstre est un scénario qui se répète dans les communautés qui accueillent la famille Beauchard; il y a toujours un membre du groupe qui se met en tête d'en devenir le chef, d'imposer un ordre aux autres (mettant finalement en acte les pulsions dictatoriales inabouties chez Jean-Christophe).

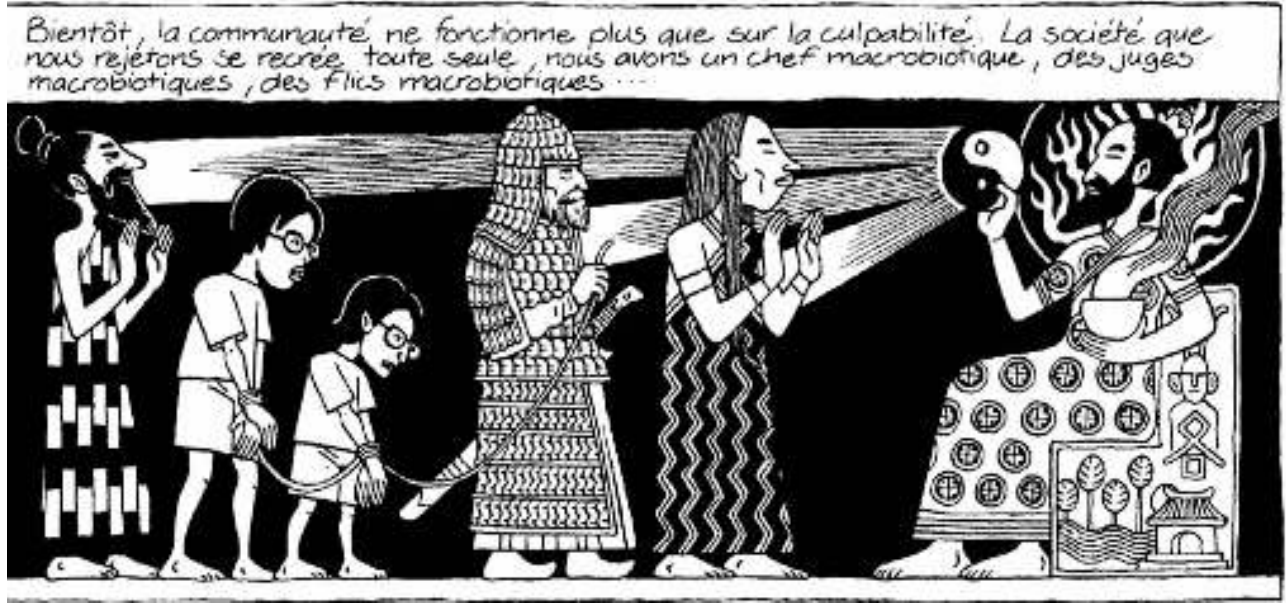

l'Ascension du Haut Mal, David B. (C) L'Association.

Or, si l'on examine de près cette transformation, on se rend compte qu'elle consiste en l'instauration d'une norme. Ce n'est pas seulement la différence entre freak et Monstre qui est effacée, c'est aussi, et plus radicalement, la dichotomie monstrueux / normal. Il ne s'agit donc pas de valoriser un "bon » monstre contre un mauvais, et d'en appeler niaisement à la tolérance de la part des garants de la norme, mais bien de contester l'idée même de norme, et de révéler que toute norme est elle-même monstrueuse. Les apprentis gourous que croise David, et dont il se moque gentiment, ne sont d'ailleurs pas si effrayants que cela, la plupart du temps inoffensifs, vite abandonnés par leurs hypothétiques fidèles, et plus dangereux pour eux-mêmes que pour autrui. Les personnages les plus inquiétants, les plus hostiles, les plus monstrueux de l'Ascension $d u$ Haut Mal, ce sont les "gens normaux ». Ainsi du médecin prêt à opérer Jean-Christophe sans se soucier des séquelles inévitables qui s'ensuivront. Ainsi également des badauds qui assistent aux crises du malade, et dont David B. dépeint magnifiquement les réactions, forcément « monstrueuses »: 

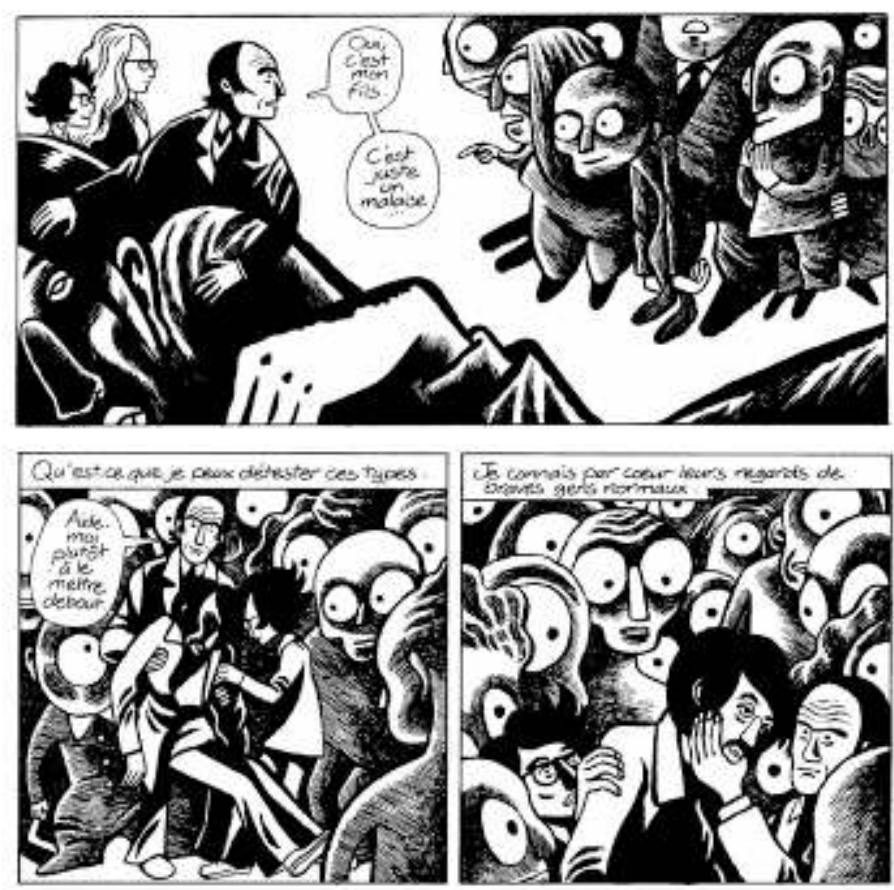

I'Ascension du Haut Mal, David B. ( L'Association.

12 Tout comme la Guerre de 14 servait de « dépucelage » à Bardamu dans le Voyage au bout de la nuit, la maladie de Jean-Christophe joue pour David le rôle de révélateur : il n'est pas de norme, il n'y a que des monstres ${ }^{10}$. Discret anarchisme de David B., dont l'idéal politique si l'on tire les conséquences de l'œuvre, indépendamment de toute prise de position éventuelle de son auteur - devrait alors consister en une "communauté des freaks» (ce qu'esquisse finalement Tod Browning dans son film).

13 Mais il ne s'agit pas pour David B. de déboucher sur un discours politique. Le geste esthétique est, lui, considérable, qui transforme, exalte, et met en question la Bande dessinée. On a dans ces lignes fortement insisté sur l'idée du "sommeil de la raison ", rencontrée à deux reprises, chez Goya, puis chez Levi ; l'expression apparaît aussi sous la plume de Jean-Jacques Rousseau, dans l'Emile (livre second) mais cette fois sans connotations péjoratives, bien au contraire, pour désigner l'enfance. Une période que Rousseau recommande de ne pas abréger trop vite, car un réveil trop brusque n'est jamais profitable.

Que diriez-vous d'un homme qui, pour mettre toute la vie à profit, ne voudrait jamais dormir? Vous diriez: Cet homme est insensé; il ne jouit pas du temps, il se l'ôte; pour fuir le sommeil, il court à la mort. Songez donc que c'est ici la même chose, et que l'enfance est le sommeil de la raison. ${ }^{11}$

14 Il y a bien chez David B. une volonté de retrouver ce bénéfique " sommeil de la raison ", qui ralentisse la « course à la mort ». L'usage de la Bande dessinée, cet art enfantin, art de freak, de voyou ${ }^{12}$, allait donc de soi pour évoquer les monstres de l'enfance : «Je ne voulais pas seulement raconter cette histoire, je voulais la raconter en dessins. ${ }^{13}$ » Manière aussi d'obéir à l'impératif baudelairien de retour à l'enfance par les moyens mêmes de la raison, seule à même de se démettre par sa propre puissance pour retrouver à volonté le regard de l'enfant sur le monde. Ce qui ne signifie en aucun cas la régression, le retour (illusoire et pathétique) à l'état d'enfance trop souvent célébré dans le neuvième art :

L'homme de génie a les nerfs solides ; l'enfant les a faibles. Chez l'un, la raison a pris une place considérable; chez l'autre, la sensibilité occupe presque tout l'être. Mais 
le génie n'est que l'enfance retrouvée à volonté, l'enfance douée maintenant, pour s'exprimer, d'organes virils et de l'esprit analytique qui lui permet d'ordonner la somme de matériaux involontairement amassée ${ }^{14}$.

17 Aussi tourmenté soit-il, que dure encore longtemps le sommeil de David B.

\section{NOTES}

1. «Un certain David B. », entretien avec Gilles Ciment et Thierry Groensteen, $9 e$ Art, $n^{\circ} 11$, dossier David B., CNBDI et Éditions de l'An 2, janvier 2005, p.60.

2. Manière, au passage, de raconter une vocation de dessinateur née dans la souffrance, et non dans l'euphorie enfantine ou adolescente pour les « p'tits miquets ».

3. Le Cheval blême, L'Association, Paris, 1992 (c'est le premier livre publié par

L'Association) ; Les Complots nocturnes, Futuropolis, 2005.

4. Entretien avec David B., op cit., p.60.

5. Comme Le Cheval blême et Les Complots nocturnes, déjà cités. Plusieurs rêves sont d'ailleurs narrés dans ce sixième tome.

6. Freaks (1932) met en scène toute une série de phénomènes de foire (nain, femme à barbe, etc.) qui se vengent d'une femme « normale » après qu'elle a manipulé l'un d'entre eux pour son argent.

7. Ibid., p. 61.

8. Ibid., p. 58.

9. Dans l'avant-propos à ses Histoires naturelles.

10. Le contexte politique n'est guère présent dans l'Ascension, qui se déroule presque uniquement dans les « marges » de la société. Le dernier tome rappelle pourtant le danger d'être un épileptique seul à Paris " sous le septennat de Giscard », où l'on peut se 
faire impunément passer à tabac, car « la police a tous les droits et la personne qui a une crise d'épilepsie dans la rue n'en a aucun », tome VI, p. 23-24.

11. J.-J. Rousseau, Émile ou l'éducation, Garnier-Flammarion, 1966, p. 132.

12. Voir dans ce même dossier, Du Voyou au Critique.

13. «Un certain David. B. », op. cit., p.58. Rousseau, précisément, quelques lignes après la citation rapportée plus haut, déclare que les enfants, " avant l'âge de raison ", ne pensent pas avec des idées, mais avec des images.

14. Charles Baudelaire, «Le peintre de la vie moderne » dans Critique d'art, Paris, Gallimard, coll. « Folio essais », 1992, p. 350.

15. Christian Rosset, « L'ascension de la Bande dessinée », $9 e$ Art, $n^{\circ} 11$, op. cit., p. 86.

\section{AUTEUR}

\section{RENAUD PASQUIER}

renaudpasquier(at)gmail.com 